\title{
Thermal Energy Storage Systems
}

Bernardo Buonomo, Luca Cirillo, Alessandra Diana, Anna di Pasqua, Davide Ercole, Vincenzo Fardella, Oronzio Manca*, Sergio Nardini

Dipartimento di Ingegneria, Università degli Studi della Campania “Luigi Vanvitelli”, Via Roma 29, 81031 Aversa (CE), Italy

Corresponding Author Email: oronzio.manca@unicampania.it

https://doi.org/10.18280/ti-ijes.640108

Received: 28 December 2019

Accepted: 15 January 2020

\section{Keywords:}

thermal energy storage, energy consumption, solar energy, sensible and latent TES

\begin{abstract}
Energy demand depend strongly on growth of world population, householders and urbanization. The energy consumption forecasting allows to give a guide for the future development in technologies. The need in energy saving as an energy source and the thermal energy storage can play a strategic role in energy recovering. After a general description of the main concept of thermal energy storage are given the classification of the different storage systems involved in several applications. It is underlined that one of the main applications is related to the solar energy conversion systems and their development is linked to the economic policy choices on energy.
\end{abstract}

\section{INTRODUCTION}

All discussions on energy demand should start from the data related to the growth of world population, householders and urbanization and their forecasting. In Figure 1, this information is reported in three different diagrams where they are given as a function of time, with an evaluation on forty years for the two first diagrams and on sixty years for the third one. Each information is subdivided in five areas: Africa, India, China, OECD and Rest of World and it is interesting observe that the households present a higher increase with respect to the population increase. Moreover, the highest increase in the urbanization is expected in China with a value in 2040 three times the one in 1980. In Figure 2, the rate of growth per year is indicated for the population, the gross domestic product (GDP) and energy demand. The highest value is related to the GDP whereas for the energy demand are given two scenarios without and with energy saving. In the second case the energy saved represents more of $40 \%$ less than the energy demand in the first scenario. In the different actions linked with the energy saving, the thermal energy storage can be included as one of the most efficient to recover thermal energy.

The forecast related to the energy needs to allow global living standards to continue the growth with the evolving transition is shown in Figure 3. In this scenario all non-fossil sources increase significantly whereas among the fossil sources the gas source presents the highest increase. This indicates a reduced increase in carbon dioxide emissions which represent one of most important problem related to the climatic variation. A projection in terms of different energy consumption scenarios and related carbon dioxide emissions is depicted in Figure 4. The forecast for the "rapid transition" underlines the significant decrease in $\mathrm{CO}_{2}$ emissions, consequent to the strong reduction in coal use as energy source. The "more energy" scenario determines a drastic increase in $\mathrm{CO}_{2}$ emissions, due to the increase in fossil fuel use. It is noted, in Figure 4 that the "evolving transition" and the "less globalization" present an about similar $\mathrm{CO}_{2}$ emissions with similar fossil fuel consumptions.

The previous analysis highlights the need in energy saving as an energy source and the thermal energy storage can play a strategic role in energy recovering.
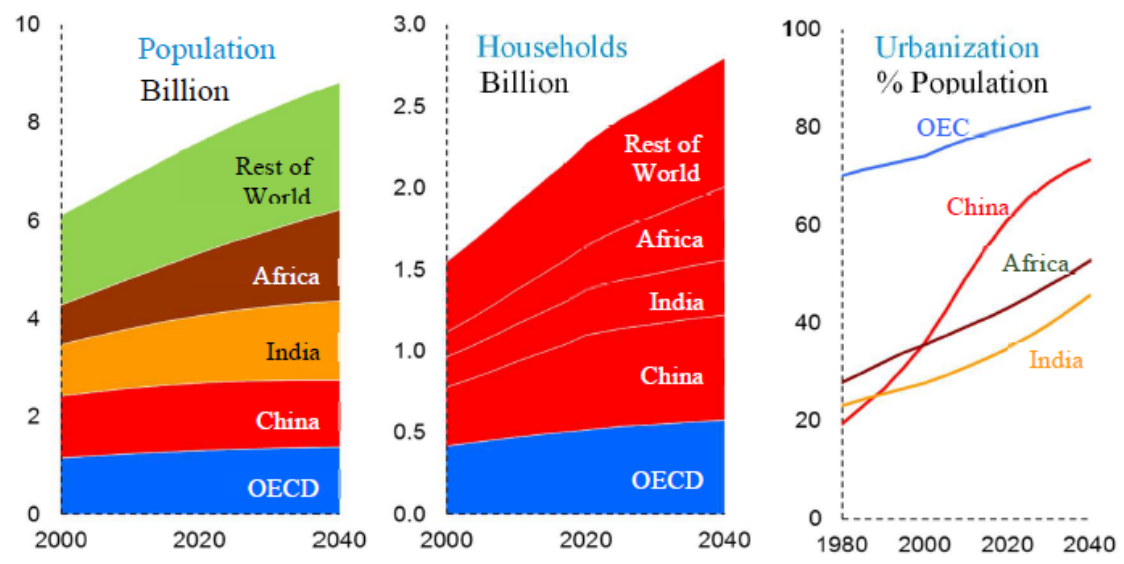

Figure 1. Energy drivers: Population, households and urbanization [1] 

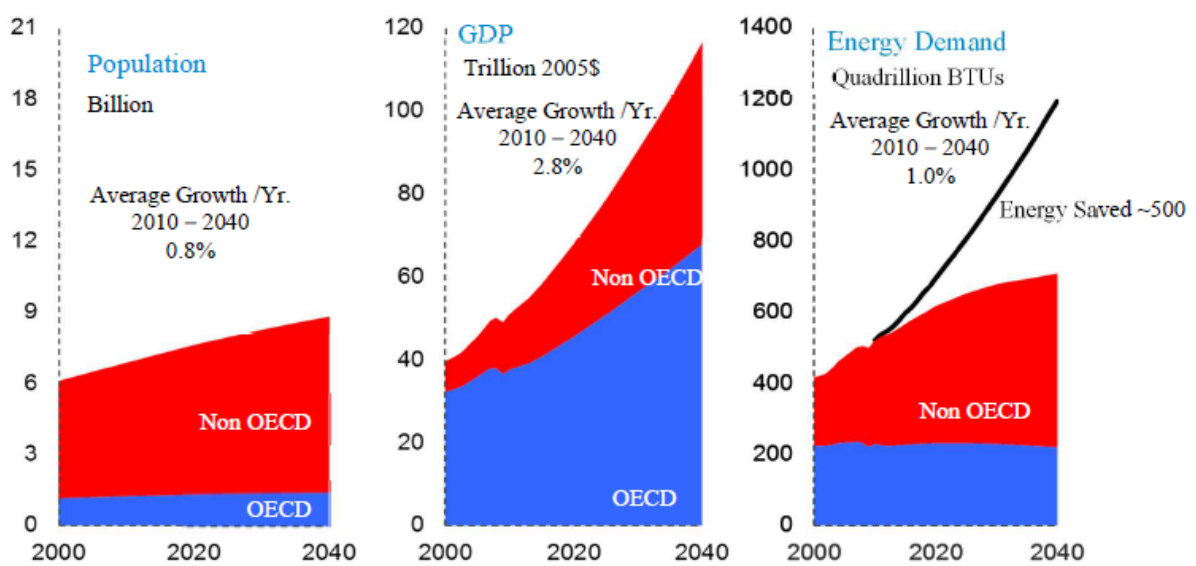

Figure 2. Forecasting of population, gross domestic product and energy demand [1]

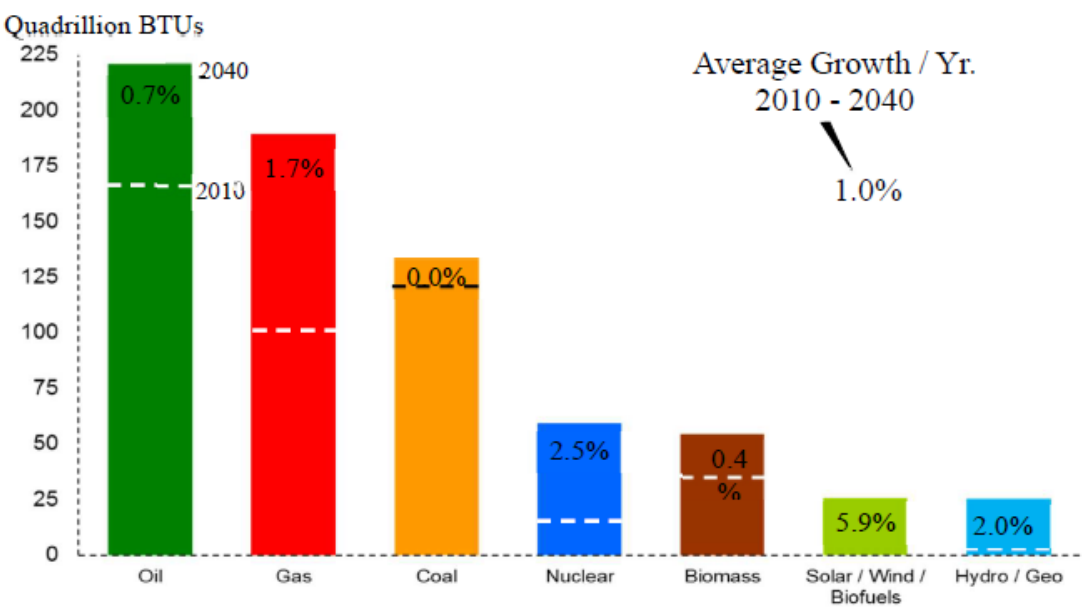

Figure 3. Forecasting of energy sources demand [1]
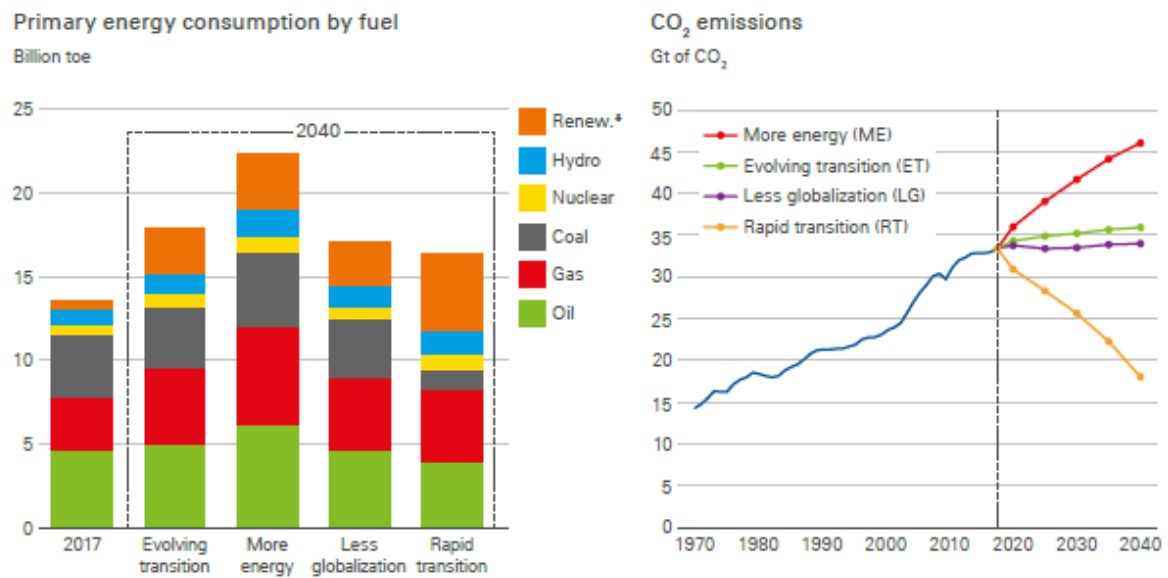

Figure 4. Forecasting of energy sources demand and carbon dioxide for four scenarios [1]

\section{GENERAL CONCEPT IN THERMAL ENERGY STORAGE}

A possible definition of energy storage is storing of some form of energy that can be drawn upon at a later time to perform some useful operation. A device that stores energy is also called accumulator. All forms of energy either potential energy (e.g. chemical or gravitational), kinetic energy, electrical energy or thermal energy, can be stored with an appropriate method, system or technology that means that every form of energy has itself an accumulator.

In Figure 5, the traditional types of energy storages such as mechanical, chemical, biological, magnetic and thermal energy storages. Some of them have different forms of energy as showed in Figure 5. Energy storage technologies are strategic and necessary components for the efficient utilization of renewable energy sources and energy conservation.

The energy conservation and management are needed in several industrial and commercial applications in order to supply thermal energy. Different systems are employed to 
satisfy the energy demand which can vary on daily, weekly and seasonal bases. The thermal energy storage is very useful for energy conservation and it allows to align energy production with consumer demand.

Thermal energy storage is expanding mainly within the renewable energy technologies and it is very important to deliver energy to eliminate or reduce the intermittence mainly in solar energy systems.

Solar energy is an important alternative energy source that will likely be utilized in the future. One of the main limiting factors in the application of solar energy is its cyclic time dependence. The solar systems require energy storage to provide energy during the night and overcast periods.

A line diagram of a typical solar energy utilization system is shown in Figure 6. The solar collector can supply directly the load, or the collected solar energy is stored and, later it is employed and increased by means an auxiliary supply, if necessary.

\section{ENERGY STORAGE SYSTEMS}

\begin{tabular}{l}
$\begin{array}{c}\text { Mechanical Energy } \\
\text { Storage }\end{array}$ \\
\hline - Hydrostorage \\
- Compressed air storage \\
- Fyraheels
\end{tabular}

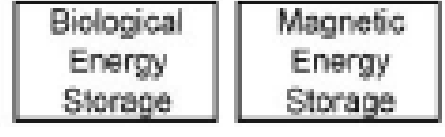

- Electrochemical batieries

- Organie molecular storage
Thermal Energy Storage

- Sensible heat storage

- Latent heat storage

- Chemical heat storage

Figure 5. Classification of energy storage systems [2]

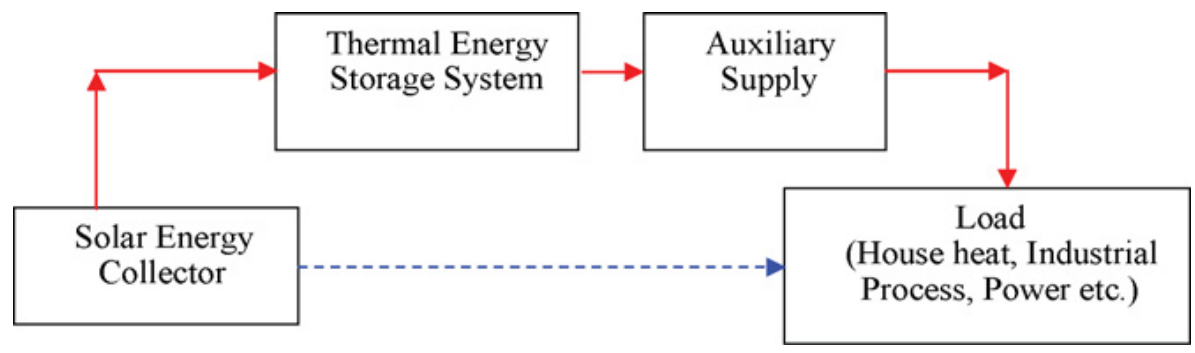

Figure 6. Solar energy system with different components and load

\section{THERMAL ENERGY STORAGE SYSTEM CLASSIFICATION}

The thermal energy storage (TES) systems have the potential of increasing the effective use of thermal energy equipment and of facilitating large-scale switching. They are useful for correcting the mismatch between the supply and demand of energy. A more detailed subdivision of TES systems is depicted in Figure 7. Thermal storage is sensible or latent.

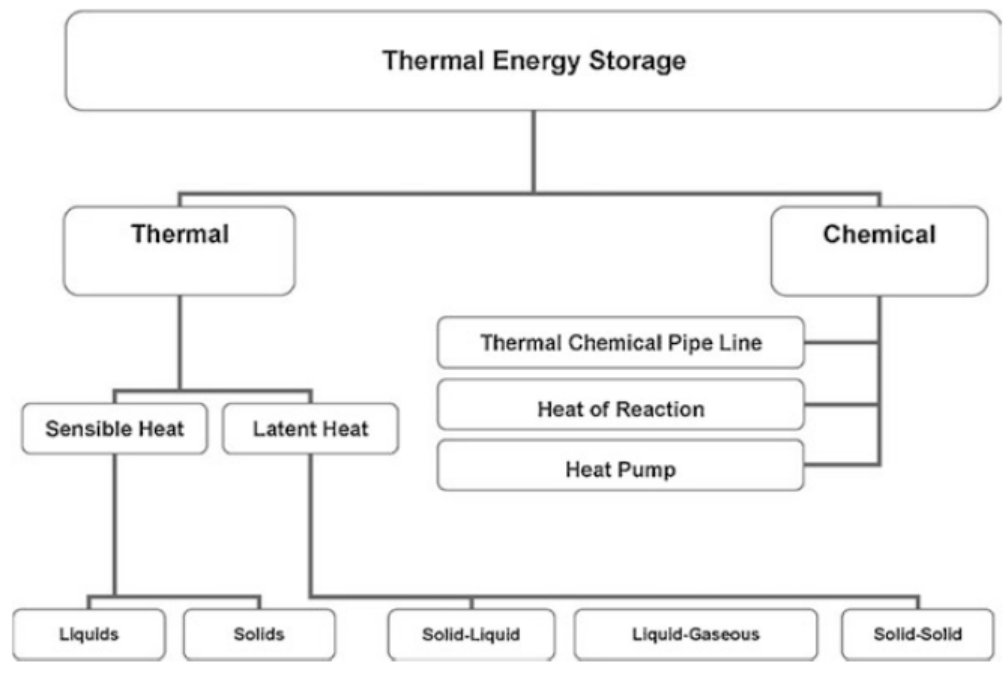

Figure 7. Different types of thermal storage of solar energy [3]

In sensible heat storage the energy is stored in the change of temperatures of substances that experience a change in internal energy. Besides the density and the specific heat of the storage material other properties are important for sensible heat storage are operational temperatures, thermal conductivity and diffusivity, vapor pressure, compatibility among materials 
stability, heat loss coefficient as a function of the surface areas to volume ratio, and cost. Sensible TES consists of a storage medium, a container and inlet/outlet devices. Tanks must both retain the storage material and prevent losses of thermal energy. Sensible heat storage can be made by solid media or liquid media. Solid media are usually used in packed beds, requiring a fluid to exchange heat.

In latent heat storage media, the energy is stored nearly isothermally in some substances as the latent heat of phase change, as heat of fusion (solid-liquid transition) or heat of vaporization (liquid-vapour transition). Mainly the solidliquid transition is used, and substances used under this technology are called phase change materials (PCM). Storage systems utilizing PCM can be reduced in size compared to single-phase sensible heating systems. Heat transfer design and media selection are more difficult, and experience with low temperature salts has shown that the performance of the materials can degrade after moderate number of freeze melt cycles.

Phase change materials allow large amounts of energy to be stored in relatively small volumes, resulting in some of the lowest storage media costs of any storage concepts.

\section{DESCRIPTION AND SOLAR ENERGY APPLICATIONS}

Several materials are employed in thermal energy storage also to improve the heat transfer behaviors in phase change materials. The porous media are the most used and they are made up by foams, packed beds and honeycomb. The material depends on the temperature range and it can be in high conductive metal for temperature up to $500-600^{\circ} \mathrm{C}$ or ceramics if the operating temperature is over $600^{\circ} \mathrm{C}$. In Figure 8 are given some examples of metal foams, packed beds and honeycomb with parallel squared channels. It should be underlined that the honeycomb systems are the more flexible because the porosity can be varied in a large range of values whereas the one for packed beds is around 0.40 and for metal foams is between 0.80 and 0.97 . Different method or solutions which depend on the main parameters in the heat storage such as heat capacity material (porous media) geometry of porous medium (spheres, foams, channels ....) working fluid (gas: air, $\mathrm{CO}_{2}$ ).

A fundamental application of thermal energy storage is in solar energy systems. They are employed for the thermal storage at high temperature solar systems $\left(>800^{\circ} \mathrm{C}\right)$ and the sensible thermal energy storage systems are used. For mean and temperature solar systems both sensible and latent thermal energy storage can be employed. Moreover, thermal energy storage systems can be used in photovoltaic system and in photovoltaic/thermal systems.

It is interesting to integrate a latent thermal storage system in ventilated roof [4-7], in Figure 9, or solar chimney [5-8], in Figure 10, or in integrated systems [7-9], as given in Figure 11.
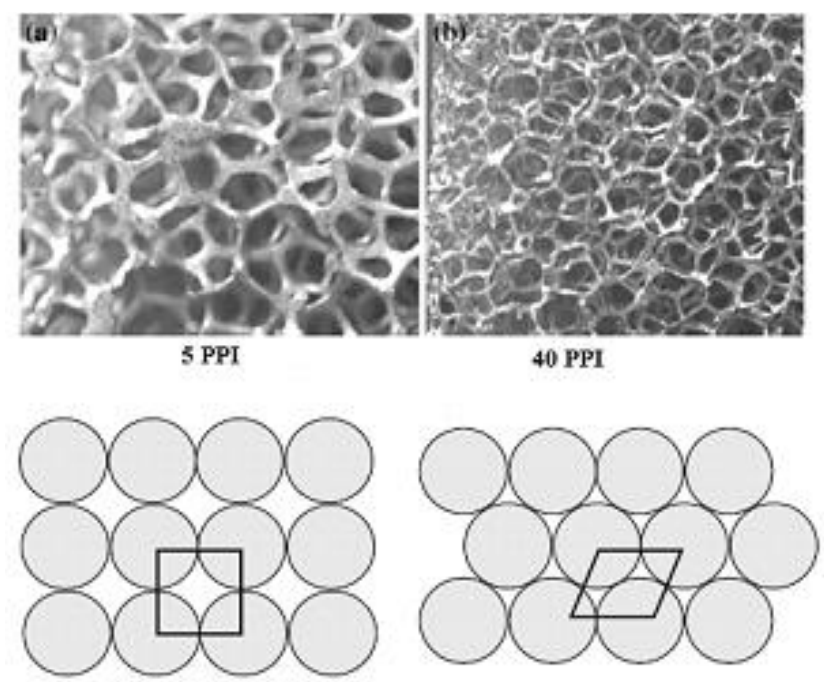

Packed bed with spherical particles a cubic packing, b thombobodral packing

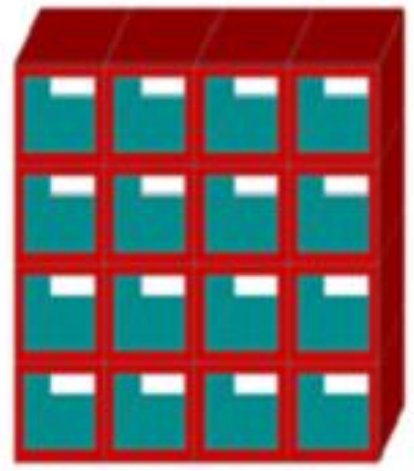

Honeycomb - parallel channels

Figure 8. Different types of porous media for thermal energy storages 


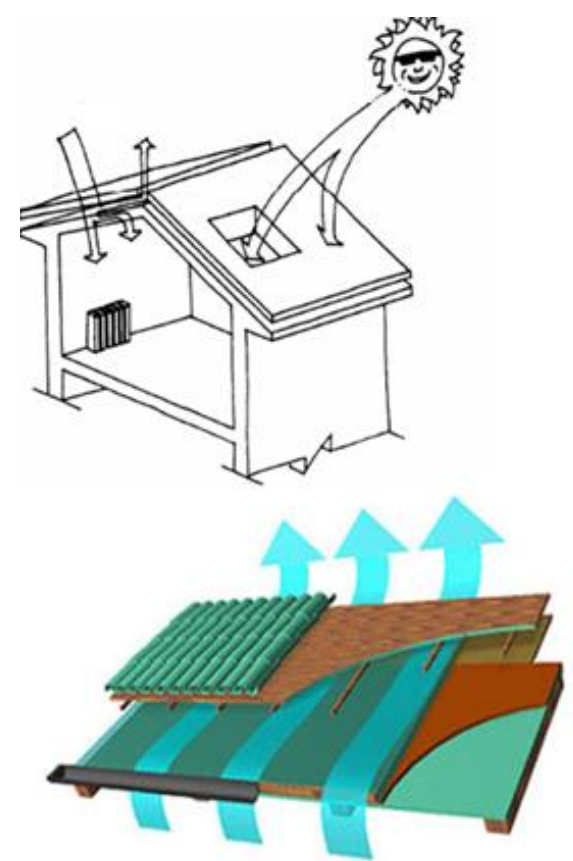

Figure 9. Ventilated roof
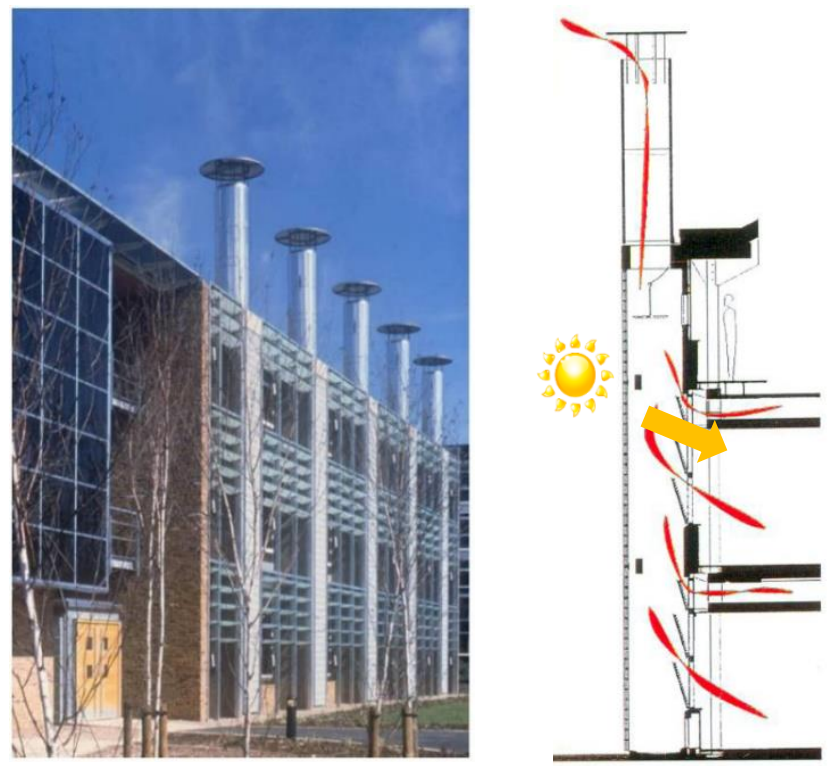

Figure 10. Solar chimney in buildings

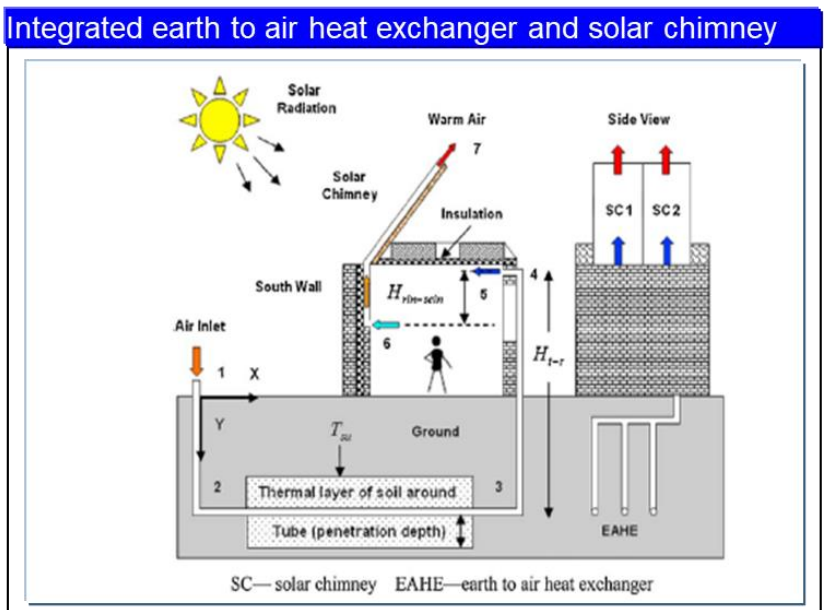

Figure 11. Integrate renewable energy systems [9]

\section{CONCLUSIONS}

The thermal energy storage can be useful in energy recovery and energy consumption reduction. It finds a fundamental use in solar energy systems and, consequently, it can play an important role in the energy transition related to the reduction of fossil fuels and carbon dioxide emissions. It should be underlined that the diffusion of solar energy technologies is also a political choice also in terms of development of the products because the solar source is free, and it can be employed by anyone. It is a democratic form of energy whereas, for example, fossil fuels and nuclear energy can be controlled by a few, leading to a possible monopoly.

It is interesting, at the end of this note to highlight the number of papers published up to 2019 and found in Scopus under the words "thermal energy storage". They are about 35000 products and the distribution for years are shown in Figure 12. It is observed that in the last ten years there has been an almost exponential growth.

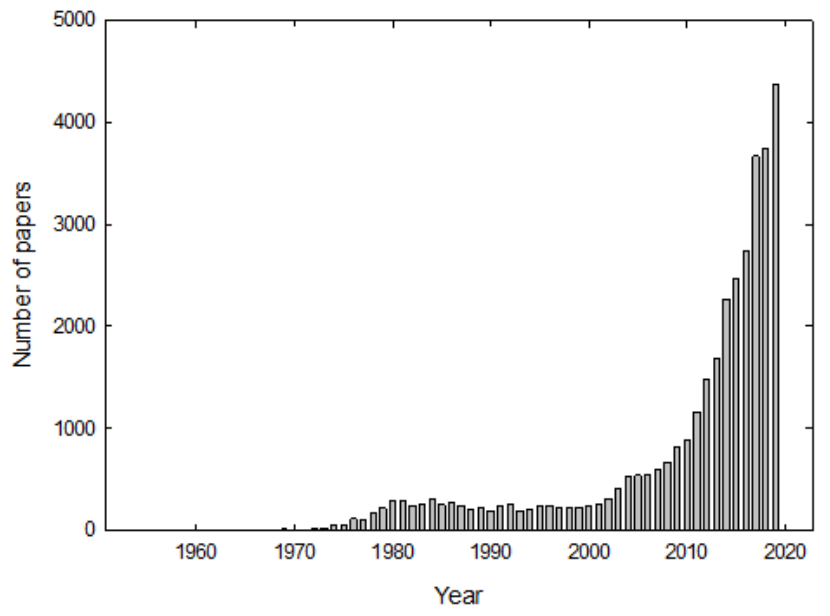

Figure 12. Published product, source Scopus 2019

\section{REFERENCES}

[1] Centre for Energy Economics Research and Policy (2019). Heriot-Watt University, Edinburgh. BP Energy Outlook, 2019 edition.

[2] Dincer, I., Rosen, M.A. (2002). Thermal energy storage, systems and applications, New York, Wiley.

[3] Sharma, A., Tyagi, V.V., Chen, C.R., Buddhi, D. (2009). Review on thermal energy storage with phase change materials and applications. Renew. Sust. Energy Rev., 13(2): 318-345. https://doi.org/10.1016/j.rser.2007.10.005

[4] Chungloo, S., Limmeechokchai, B. (2009). Utilization of cool ceiling with roof solar chimney in Thailand: The experimental and numerical analysis. Renewable Energy, 34(3): 623-633. https://doi.org/10.1016/j.renene.2008.05.026.

[5] Zhai, X.Q., Song, Z.P., Wang, R.Z. (2011). A review for the applications of solar chimneys in buildings. Renewable and Sustainable Energy Reviews, 15(8): 3757-3767. https://doi.org/10.1016/j.rser.2011.07.013

[6] Shia, L., Zhang, G., Yang, W., Huang, D., Cheng, X., Setunge, S. (2018). Determining the influencing factors on the performance of solar chimney in buildings. Renew. Sust. Energy Rev., 88: 223-238. 
https://doi.org/10.1016/j.rser.2018.02.033

[7] Monghasemi, N., Vadiee, A. (2018). A review of solar chimney integrated systems for space heating and cooling. Application. Renew. Sust. Energy Rev., 81: 2714-2730. https://doi.org/10.1016/j.rser.2017.06.078

[8] Kimouche, N., Mahri, Z., Abidi-Saad, A., Popa, C., Polidori, G., Maalouf, C. (2017). Effect of inclination angle of the adiabatic wall in asymmetrically heated channel on natural convection: Application to doubleskin façade design. J. Build. Eng., 12: 171-177. https://doi.org/10.1016/j.jobe.2017.06.002

[9] Maerefat, M., Haghighi, A.P. (2010). Passive cooling of buildings by using integrated earth to air heat exchanger and solar chimney. Renew. Energy, 35: 2316-2324. https://doi.org/10.1016/j.renene.2010.03.003 